\title{
Software with Biofeedback to Assist People with Down Syndrome
}

\author{
D. Lima \\ PUC Goiás \\ Av. Universitária \\ 1440 Goiânia/GO, \\ Brazil
}

\author{
M. Sousa \\ PUC Goiás \\ Av. Universitária \\ 1440 Goiânia/GO, \\ Brazil
}

\author{
R. Araújo \\ PUC Goiás \\ Av. Universitária \\ 1440 Goiânia/GO, \\ Brazil
}

\author{
J. Hannum \\ PUC Goiás \\ Av. Universitária \\ 1440 Goiânia/GO, \\ Brazil
}

\author{
A. da Rocha \\ UnB-Gama \\ Área Especial, \\ Projeção A, \\ UnB - Setor Leste, \\ Gama, Brazil
}

\author{
T. Barbosa \\ PUC Goiás \\ Av. Universitária 1440 \\ Goiânia/GO, Brazil
}

\begin{abstract}
Down syndrome is caused by the presence of three chromosomes 21 in all or most cells of a person [1]. A person with Down syndrome has different chronological and functional age, so the same response is not expected as those without the syndrome [2]. This deficiency stems from brain damage and functional imbalances of the nervous system, resulting in some learning difficulties, which can vary from basic literacy to performing daily activities. The applications developed in this work are intended to help these people in their literacy, while monitoring their attention levels and detecting their expressions. The assistance is provided through activities such as pairing vowels and matching words with corresponding images and sounds. On the other hand, a biofeedback algorithm called Attention Meter runs in parallel with the activities, monitoring the user's attention during the execution. This algorithm is implemented as a framework that can be used by any application running Android or RemixOS. Finally, a performance report of the student engagement and learning is generated for a professional analysis, according to the attention level.
\end{abstract}

\section{General Terms}

Down syndrome, Android, Java, Software

\section{Keywords}

Biofeedback, OpenCV, Attention, Activity, Framework

\section{INTRODUCTION}

An affective game is a term derived from the concept of Affective Computing, established by Rosalind Picard from MIT, in the 90's. While playing an affective game, the player indirectly interacts with it through electrophysiological signals [3]. After that, some information about the emotional state of the player can be extracted from these signals, such as fright, fear or anxiety. In this project the term biofeedback is adopted referring to functionalities that enable the implementation of Affective Gamming.

There are initiatives in software development designed to help the learning process of people with Down syndrome. Participar [4], Livox [5], vSked [6] and Mobile Communication Tools (MOCOTO) [6] are examples of those.
These projects were designed for rehab through game activities, which enable an increase in interaction and engagement of the users.

The projects Participar and Livox are meant to assist the literacy process of their users, through activities using music, for example. On the other hand, the vSked and MOCOTO projects seek to offer activities that automate the PECS (Picture Exchange Communication System). The PECS is a method intended to help people who don't own verbal communication, by using picture exchanging.

All the mentioned applications are designed to support people with cognitive development delay. However, none of them use biofeedback resources to verify or validate the results, which is the main contribution of this work. The biofeedback component is implemented using a webcam to verify the user's attention level during the software usage.

There is a project called Alfadown in Pontifical Catholic University of Goiás (PUC-GO) which supports students with Down syndrome. It's divided into 2 complexity levels: rudimentary, that covers people with basic literacy need, and advanced, that deals with already literate people, seeking to hone their skills.

This work consists in the development of an Android application that uses Affective Computing functionalities. The application uses virtual activities to help the Alfadown students in their learning process. The activity execution is monitored by a biofeedback algorithm that uses the device's camera as a sensor, which can automatically assist, monitor and evaluate the rehabilitation activities, generating a report to be analyzed by the professionals who go along with the students.

\section{MATERIALS AND METHODS 2.1 Technology}

This application is designed to be device independent. It was developed using the Android platform, and it can run in any Android 4.0.4 or superior compatible device, including PCs running a Remix OS. The Android operational system was chosen as a platform due to the large number of compatible 
devices and their reduced costs, enabling an enlargement in the access of this technology.

The development of the activities was made using Google's Integrated Development Environment (IDE) Android Studio [7] and the tests were made in several devices: Compute Stick (Fig. 1 (a)), The Acer Tablet A500(Fig. 1(b)), a PC running Remix OS, and an Intel also running Remix OS (Fig. 1(c)).

The biofeedback module was developed for the Android platform devices using the Android SDK and features available in the Native Development Kit (NDK). Java and $\mathrm{C}++$ were used as programming languages, while NDK allowed the implementation of parts of the framework to run in native code languages such as $\mathrm{C}$ and $\mathrm{C}++$.

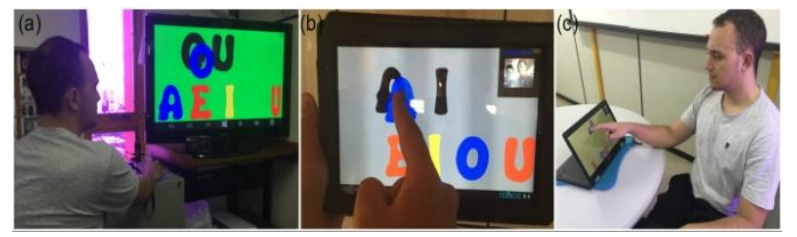

Figure 1. Application being executed in different devices: (a) Compute Stick, (b) Tablet Acer Iconia A500, (c) Remix OS

In order to work with image manipulation, the Open Source Computer Vision (OpenCV) multiplatform library was used. OpenCV is released on a BSD license, free for academic and commercial use. OpenCV implements a variety of image interpretation tools, ranging from simple operations, like a noise filter, to more complex operations, such as motion analysis, pattern recognition, and 3D reconstruction.

The input is mainly the image stream obtained in real time through the camera sensor of the mobile device. The camera must be properly positioned so that the targeted individual can almost directly look at it. The framework is also capable of analyzing videos or static images provided to the software.

\subsection{Specifications and Prototypes}

\subsubsection{Customization}

Students at the rudimentary level have learning challenges, such as letter pronunciation and recognition, and also reading/writing problems. In advanced level, some students have difficulties to match the output sound with the corresponding image. Thus, the application should offer customizable activities; since each student has particular issues, the monitors can set the appropriate parameterization for each of their individual pupils. In order to do that, a configuration screen was created. In this screen, the monitor can set several parameters, such as the interaction mode that can be done by touching or dragging elements on the screen, the number of interactions before finishing an activity, as well as attention meter settings like the camera to be used and the resolution, along with some other settings. In addition, the application offers integration with the Google Drive's cloud service, allowing these settings to be done remotely.

\subsubsection{Activities:}

The application has 4 activities; Activities1 to 3 are based on combining alphabet letters to form words, and the 4th one is based on sensorial stimulation, corresponding sound effects with their respective image and names.

\subsubsection{Interface:}

The activities use both sounds and images to create interactions. The importance of using sounds and images is emphasized by Pivetta [8], since people with Down syndrome may have auditory loss, which makes the hearing harder. Also, according to Leite and Baptista [9], they need concrete and visual stimuli that make part of the daily life. The 4th activity uses that premise by adding media from everyday situation, such as rain sounds, claps, car engines and alarm clocks. Besides images and sounds, vivid colors and large fonts for the text were used to ease the visualization, as proposed in the app developed by Herrera, Dickie and Schulenburg [10].

Moreover, people with Down syndrome need more time than usual to learn [8], which makes important the use of repetitions during the process. It is used in the activities, and the number of these repetitions can be manually set by the monitor. Furthermore, according to Martin \& Pear [11], a positive reinforcement is something presented after a behavior that increases the probability of this behavior to happen again. It is used in the activities through scores and a virtual pet, named Lori that congrats the student after every success.

People with Down syndrome usually have hypotonia, (muscle flaccidity), which may spoil their motor skills [8]. However, this harm can be healed practicing. Thereby dragging the images around the screen can be a good exercise for this. Fig. 2 shows the interface of the 4 th activity running.

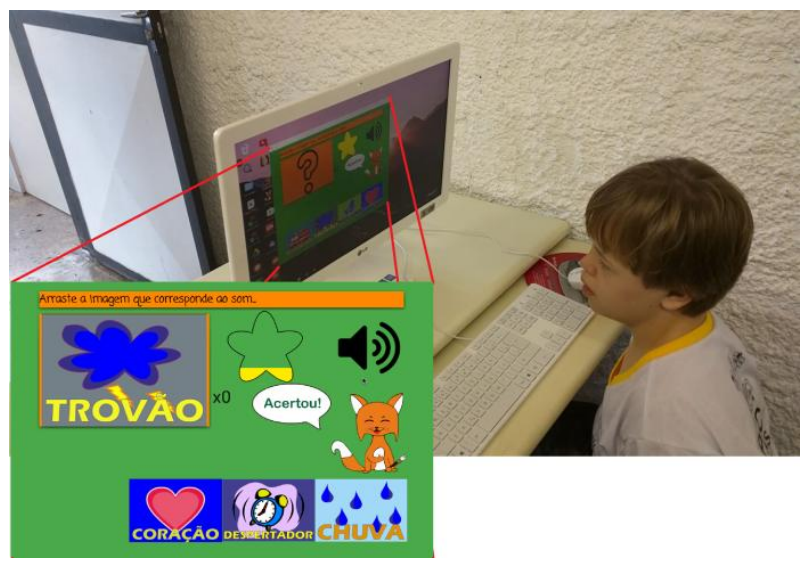

Figure 2. Screen capture of the 4th activity (Portuguese). In English: Title (Drag the sound corresponding image), Trovão (thunder), Coração (heart), Despertador (alarm clock), Chuva (rain), Acertou (correct)

\subsection{Biofeedback}

\subsubsection{Structural Description:}

The biofeedback algorithm used in this work is called Attention Meter, a framework developed for the Android system by Edson Douglas [12], based on the work of C. J. Lee[13].

A framework is a set of classes that constitutes an abstract project for the solution to a family of problems [14]. The Framework Structure can be seen in the class diagram shown in Fig. 3, which contains information about the methods, attributes, function names, and how they were integrated into the system.

The abstract class CameraCallBack defines an application template enforcing the classes that extend it to implement their methods and the contracts defined in the CvCameraViewListener interface. These methods are responsible for configuring and retrieving the data from the device's camera. Likewise, if it is necessary to create a new 
class to receive data from the camera, the class CameraCallBack just needs to be extended, the same way it is done in the class FaceDetect, which is responsible to identify the face, set the attention level and identify the user's emotion.

In this relationship, the concepts of inheritance and polymorphism from object-oriented programming are applied. These concepts allow attributes and methods to be shared by classes and implemented in their own way, making it easier to insert new features that also analyses the data obtained from the camera.

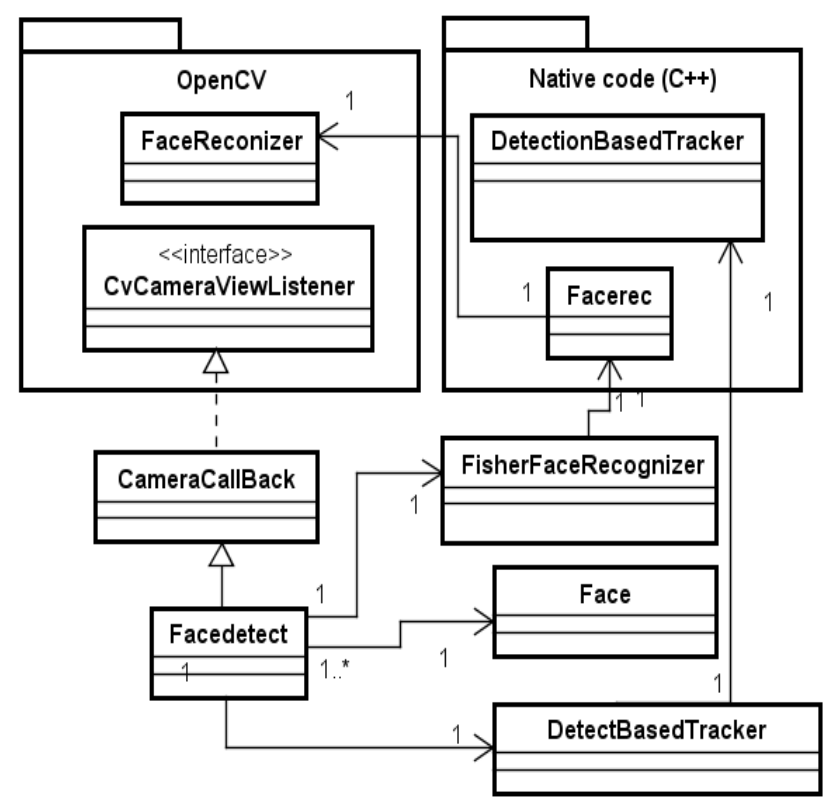

Figure 3. Class Diagram that describes the structure of the proposed Framework

\subsubsection{Attention Monitoring}

This algorithm measures the attention level of the users by analyzing the variations in their facial micro expressions. It can be executed in real time locally while the students are doing the activity or remotely, recording the faces and applying the algorithm any time after finishing. Fig. 4 shows the algorithm running in real time during one of the activities.

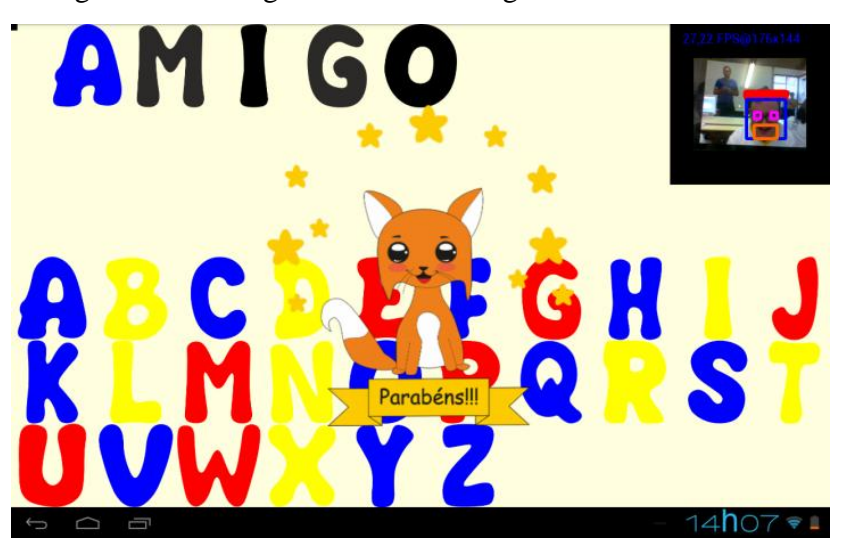

Figure 4. Screen capture of the 3rd activity running the biofeedback algorithm in Portuguese. In English: Amigo (friend), Parabéns (congratulations)

There is an attention meter developed at the MIT Media Lab [15] that aims to measure a user's attention level. It has been adapted for the android platform and included in the composition of the framework. The software is able to identify a person and start to measure the attention level of the individual, as long as they look towards the camera. Each frame obtained either by using a saved video or in real time through the camera, a face detection algorithm described below is applied in order to determine the attention level.

In order to determine the attention level, the software needs to firstly identify the face present in each frame. The face detection method proposed by Paul Viola and Michael Jones [16] present in the OpenCV library is used. This method utilizes a detection technique based on the appearance of the object that when used together with some key features allow a fast and robust implementation of facial detection. These methods tend to learn features from training with positive images (images with faces) and negative images (images without faces), to later detect objects in other images.

Using facial knowledge and looking for distinctive brightness gradients of the eye the attention meter can quickly find, detect eyes, and measure the intermittent rate of a face over several frames. Each identified face is given a rating for attention that varies over time. It starts at 0 and increases according to the attention exhibited by the individual.

Combining affection, emotion, movement patterns, small head gestures, and the score of attention in various ways, will allow us to determine high-level activities about the relationship between individuals and the target of attention. In this way, the attention meter can go beyond simply measuring attention, it can describe the relationships that individuals have with the target and other individuals.

The Fig. 5 shows the flowchart that describes the operation of the attention monitor and the Fig. 6 shows the face identification and the attention meter applied in the software to monitor real time attention of those looking towards the camera.

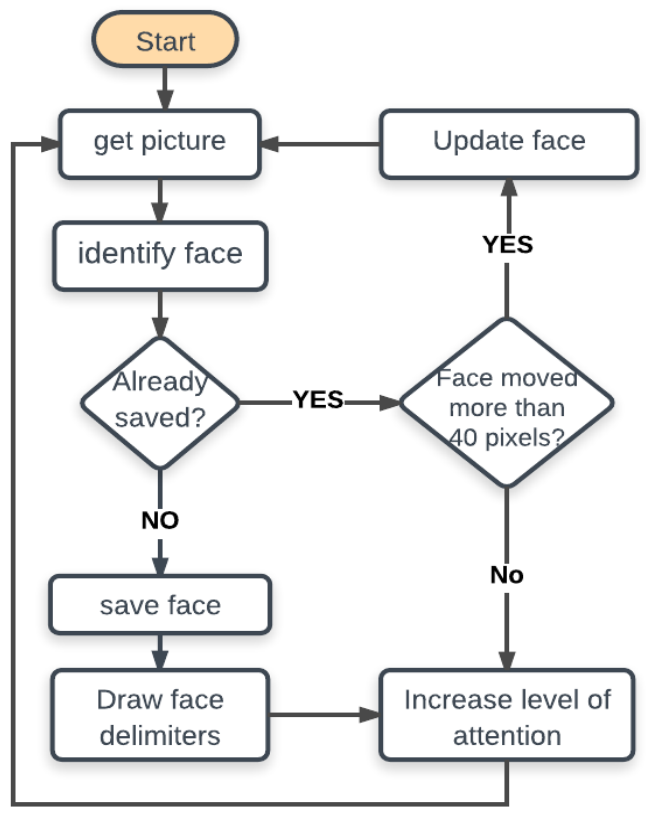

Figure 5. Flowchart that describes each step of the attention meter algorithm

\subsubsection{Expression Recognition:}

The Fisherfaces algorithm available in the OpenCV library was used in the system so it could learn to recognize expression patterns, which uses ideal machine learning for facial recognition. In Fisherfaces, all pixels of a face or the 
image that contains a face are utilized as input of the recognition system [17]. Thus, all images information can be considered in this approach despite having a disadvantage of the high dimensional data increasing computational costs. To solve this problem, the statistical method Principal Component Analysis (PCA) is used in order to have a Dimensionality Reduction.

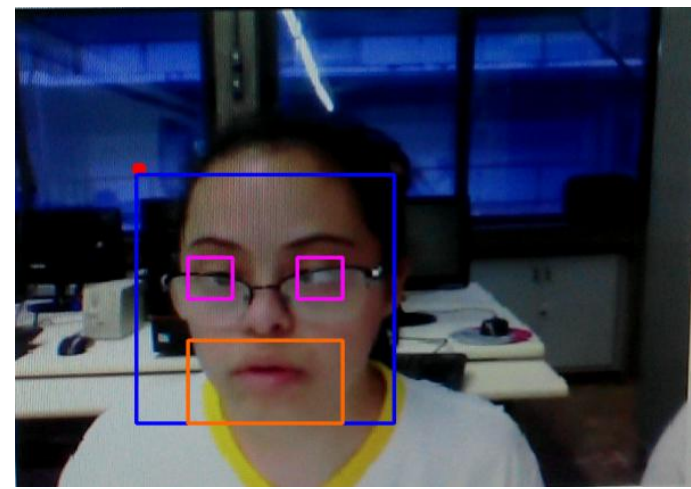

Figure 6. Shows the face delimiters drawn from the data received from the OpenCV classifier. The red bar above the face delimiter represents the current level of attention of the individual

The creation of the dataset is a very important factor in order to be successful in the recognition process. In this step, one or more face images are grouped and encoded for use in the model forming the face-space. After selecting a face and establishing a similarity of it with the dataset, the algorithm looks for features that define the face.

In this project, the dataset of images was obtained from videos collected with the help of the Alfadown researchers. The videos were recorded during the rehabilitation activities performed by the group. From the analysis of these videos, it was possible to obtain images that contained certain expressions of emotions. It was important to guarantee that the videos were recorded while the individuals performed familiar activities and suffered no interruption during it.

Expression recognition is a delicate process that is vulnerable to small changes in lighting condition. To help to minimize these problems and increase its accuracy, the images are automatically adjusted before applying the algorithm. The methods applied are conversion to gray scale, histogram equalization, and bilateral filter.

Face detection has a better performance on grayscale images, thus each image obtained is converted to grayscale. Generally, grayscale images are more robust to variations in lighting [18].

An image histogram contains the information on the number of times the hue of a color is repeated. The histogram equalization is used to adjust the intensity of the image improving its brightness and contrast, useful for getting more details in images with backgrounds and foregrounds that can be both bright or both dark [19].

The bilateral filter helps to smooth the lower contrast areas of the image without affecting the areas of higher contrast, reducing the noise present in the image while preserving its contours.

Fig. 7 shows an original image taken from the dataset, and the result after multiple adjustments has been made.
For the algorithm to begin the identification of expressions, it needs to be trained with a set of face images created from the Alfadown videos. The training set helps to establish a knowledge base with information that will be used for comparison between input faces.
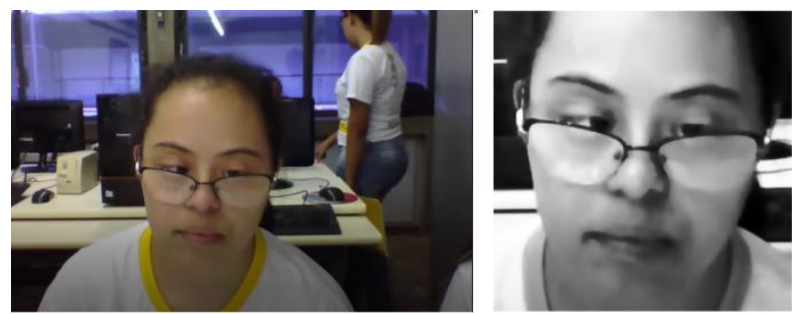

Figure 7. Before and after image of the adjustments made to increase the accuracy of the emotion identification algorithm

The system loads images with a number that represents which emotion it belongs to. Then the input image is passed to the software where the identification is made. The input face is compared to the other images in the database. Fisherfaces creates a low-level representation of the face, result from the linear discriminant analysis, over the projection of the image in the sub-space created by the PCA. Because of this comparison, the method returns a number that represents which emotion the face is associated with.

Fig. 8 shows the emotion recognition applied in a video obtained by Alfadown during the rehabilitation activities.

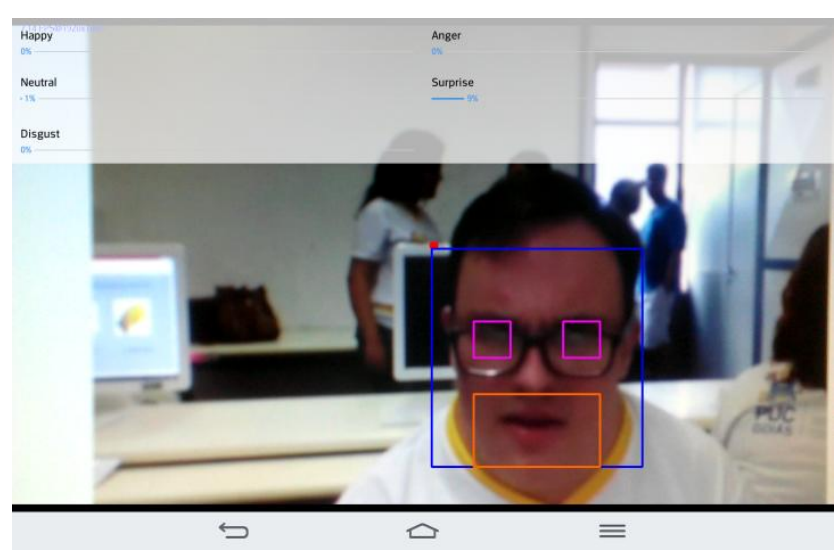

Figure 8. Emotion recognition

Finally, after applying the biofeedback algorithm, a performance report of each user after finishing the proposed activity is generated. The report contains information about the emotional state of the student during the execution. It tells to the monitors how their students did while performing that activity.

\section{USABILITY TESTS}

The usability tests follow a test script, which consists in a set of tasks such as a software evaluation quiz and definition of the test parameters. This script was made to allow the software application on the students, which was approved by the local Ethical Committee under the Presentation Certificate for Ethical Appreciation: 32702 114.2.0000.0037.

They were possible after finishing the software prototype. The tests were made using the Alfadown project computers running native Android through Remix OS, initialized using a bootable flash drive. 


\subsection{Software Evaluation Quiz}

The content of the software evaluation quiz was defined by the project members, in Portuguese, through group meetings. The defined questions allowed the developers to perform a preliminary evaluation of the software utility. Fig. 9 shows a final version of a software evaluation quiz.

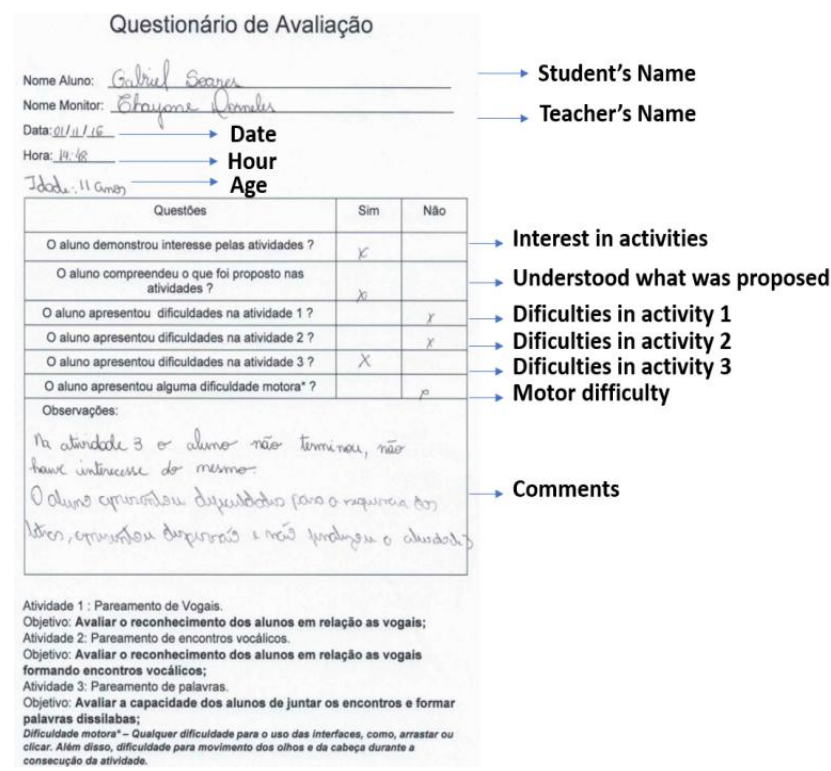

Figure 9. Software evaluation quiz. In the comments section: "In activity 3 , the student didn't finish. He wasn't interested. The student showed difficulties in the letter sequency, dispersed and didn't finish the acivity."

\subsection{Software Test Application}

The goal of each activity is specific. In the vowel matching, the objective is to evaluate the vowel recognition ability of the students. In the activity of pairing vowel encounters, the objective is to evaluate the student's recognition of the vowels making up encounters. In the word pairing activity, the objective is to evaluate the student's capacity of bringing vowel encounters together to form dissyllable words. In the sound matching activity, the objective is to evaluate the sensorial ability of the student to match images, sounds and words.

Then, the tests with the activities were made with the Alfadown students after defining the questions on the quiz. Along with the questions, some parameters were defined, such as, the number of repetitions ( 5 for activities 1, 2 and 3 and 3 for activity 4), the interaction mode (dragging), the camera to be used and the resolution of the recorded videos.

After finishing the tests, the monitors must fill the software evaluation quiz referent to the performance of that student. The quizzes are then collect to posteriori evaluation of the results. Fig. 10 shows the test application process diagram.

\subsection{Biofeedback Training}

Initially the algorithm was trained using only $80 \%$ from a database of 240 images. From these images, 48 were used for each of the five emotions. After training the algorithm, the remaining $20 \%$ of the database, was used to test the efficiency of the algorithm. These images consist in a total of 60 units, 12 for each emotion.

After providing the content for the algorithm training, the 60 remaining images were passed one by one to the algorithm to perform the analysis. Every performed analysis saved the emotion to which image it belongs to, along with the emotion that the algorithm has identified, which made it possible to identify the number of errors and correct answers.

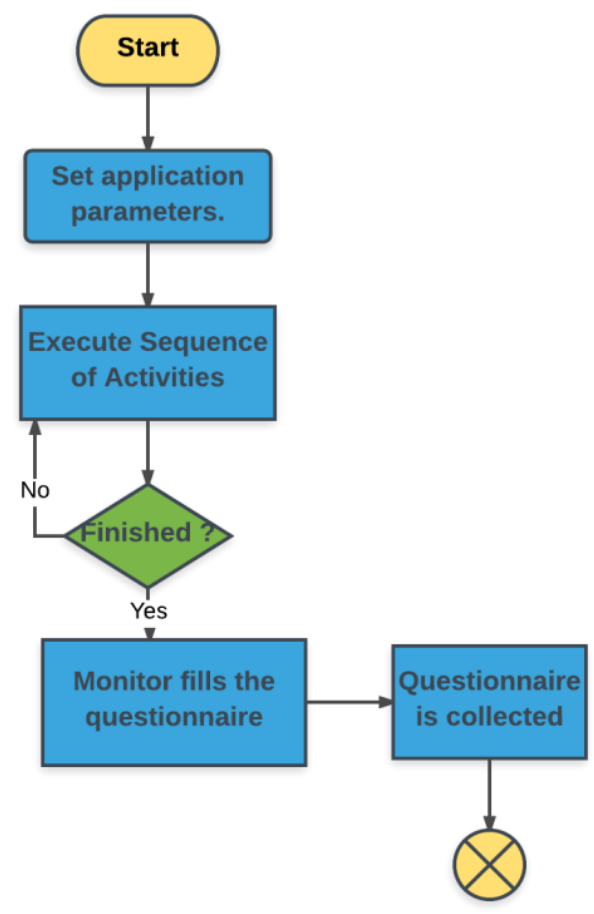

\section{RESULTS AND REVIEWS}

\subsection{Testing Results}

After collecting the reports, the statistical analysis of the data started. After calculating statistical variables and probability values, a binomial distribution was use to make inferences about the data obtained from the questionnaires. Binomial distribution is associated to situations that involve only two possible results: success or failure. Therefore, a set number of trials is verified, having only two possibilities: success or failure, where the success probability is the same for each one of the trials, that are independents.

Fig. 11 and Fig. 12 show the probabilities calculated using the data from the tests. According to the data, $93.33 \%$ of the students had shown comprehension about what was proposed in the activity (Fig. 11(a)). Similarly, $86.66 \%$ had shown interest in executing the activities (Fig. 11(c)). Thus, not all of the students that comprehended what was proposed have felt themselves comfortable or interested.

Furthermore, $33.33 \%$ of the students had shown some motor difficulty using devices like mice or dragging components on the screen during the tests Fig. 11(b). Due to this an option to match the components by clicking then instead of dragging was implemented.

Moreover, a linear increasing of the students' difficult while doing the activities 1 to 3 was noticed during the tests. It means that students show a smaller percentage of problems while doing vowel matching in the 1st activity and a bigger percentage of problems while doing word matching in the 3rd activity. This linear behavior along with the equation that represents the linear trend can be seen in Fig. 12. 
(a)

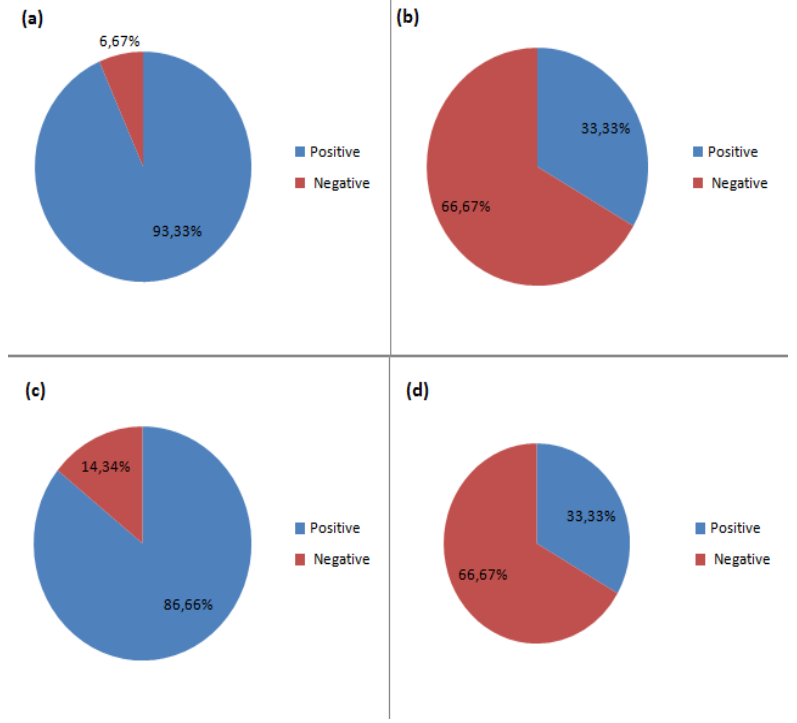

Figure 11. Calculated probabilities of the collected data. (a) Students' comprehension about the proposed activity. (b) Difficulty in activity 4. (c) Students' interest shown during activities. (d) Students that show motor skills difficulties.

The 4th activity proposed in the application has a different proposal than the others. The 1 st three activities are focused on literacy, while the 4th one is focused on sensorial stimulation. Among the performed tests, $33.33 \%$ of the students had shown some difficulty while doing the activity (Fig. 11(d)). These challenges can be difficulties while listening or identifying the sounds, probably due the ambient noise while the tests.

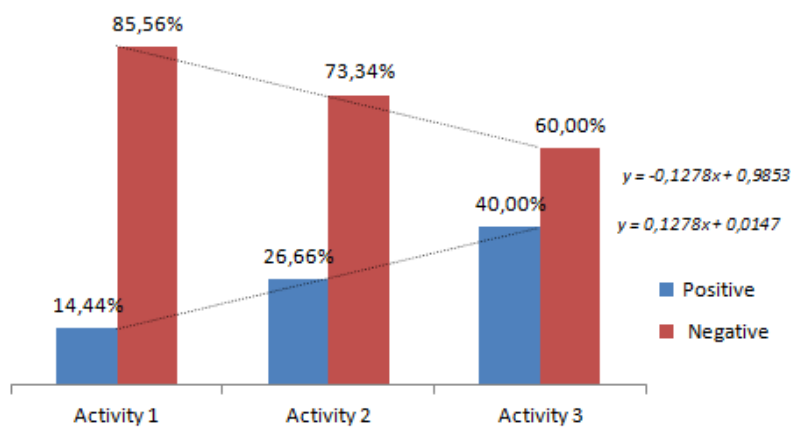

Figure 12. Probability of the difficulties shown by the students in activities 1, 2 and 3 along with the linear trend equations.

\subsection{Biofeedback Algorithm Results}

From a total of 60 images the algorithm was able to correctly recognize 40 images and miss another 20 images, obtaining a success of $66 \%$. It was possible to observe that most of the errors occurred between disgust and anger, often the expression of anger and disgust are not as clear and noticeable as other emotions such as happiness or surprise, leaving the algorithm confusing that ends up determining a wrong value for the emotion. One solution to this problem would be to improve the database by adding images of anger emotion in which the individuals are more expressive, but this would cause a difficulty in identifying more subtle gestures. Table 1 shows the confusion matrix that summarizes the results of the algorithm tests for this analysis; all correct guessing is located on the main diagonal of the table.
The numbers in the Accuracy (ACC) column show the accuracy of the classification, the images are correctly classified according to the class that belongs.

The figures in the Reliability (REL) line show the reliability of the classes in the classified image it is the fraction of correctly classified images in relation to all images classified with this class.

Table 1. Emotion recognition results

\begin{tabular}{|l|l|l|l|l|l|l|l|}
\cline { 2 - 8 } \multicolumn{2}{c|}{} & \multicolumn{7}{c|}{ Classification Results } \\
\cline { 2 - 9 } \multicolumn{2}{c|}{} & Neutral & Happy & Anger & Disgust & Surprise & ACC \\
\hline \multirow{3}{*}{} & Neutral & 11 & 0 & 1 & 0 & 0 & 0,917 \\
\cline { 2 - 9 } & Happy & 1 & 10 & 1 & 0 & 0 & 0,833 \\
\cline { 2 - 8 } & Anger & 3 & 1 & 7 & 1 & 0 & 0,583 \\
\cline { 2 - 8 } & Disgust & 6 & 0 & 4 & 2 & 0 & 0,167 \\
\cline { 2 - 8 } & Surprise & 2 & 0 & 0 & 0 & 10 & 0,833 \\
\cline { 2 - 8 } & REL & 0,478 & 0,909 & 0,538 & 0,667 & 1,000 & \\
\hline
\end{tabular}

\section{CONCLUSION}

People with Down syndrome have a number of challenges in their learning process when compared to individuals with typical learning. They take more time absorbing the content to learn [8], which can be softened by using repetitive and interactive activities. They also need visual and concrete stimuli, such as daily life elements [9], and they work better with positive reinforcement [11]. This work will be a great addition to projects like Alfadown, enhancing the endeavor of the instructors and helping Down students to surpass these difficulties, through virtual activities with plenty of audiovisual stimuli.

In Addition, the biofeedback component will help the instructors and health professionals to assist the development of each student individually by analyzing their behavior and focus during the activity. This assistance will be possible either in real time or remotely, due to the Google Drive connection, which will enable the professionals to configure the application and store the results remotely.

Some technical benefits where achieved for this project, like device independency, enabling the application to run on any PC with Remix OS or tablet with Android O.S. (version4.0.4 or superior), connection with Google Drive platform, allowing remote connection and modularized development, facilitating the addition of new features and activities.

Finally, for future updates, new activities may be added to the application, along with new features, such as voice recognition and generation of content using the student's voice, new tests may be done, monitoring the development of the students using the activity through time, and last but not least, the monitoring process of the biofeedback algorithm may be automated, enabling the application to reset its settings according to the development of each individual.

\section{ACKNOWLEDGMENT}

Special thanks to the crew of the project Alfadown, that supported this work since the beginning, especially the students Beatriz Mendes de Souza, Jessica Santillo and Michelle Oliveira Cruz, who dedicated their time and talent to this work. Also to the project advisor, Professor Talles Marcelo G. de A. Barbosa and professors Juliana Santos de 
Souza Hannum and José Olímpio Ferreira, that assisted and oriented all the development process.

This study was supported by FAPEG (in Portuguese, Fundação de Amparo à Pesquisa de Goiás) at Pontifical Catholic University of Goiás.

\section{REFERENCES}

[1] O que é? Síndrome de Down. Online: http://www.movimentodown.org.br/sindrome-dedown/o-que- e/.

[2] Bissoto ML. Desenvolvimento Cognitivo e o Processo de Aprendizagem do Portador de Síndrome de Down: Revelando Concepções e Perspectivas Educacionais. Ciências e Cognição, 200.

[3] Picard Rosalind W., Affective Computing in Cambridge: MIT press, Vol. 252. 1997.

[4] Projeto Participar, Expressar. Online: http://www.projetoparticipar.unb.br/expressar.

[5] Livox, Livox. Online: http://www.livox.com.br/.

[6] Hayed G.R, Hirano S., Monibi M., Nguyen D.H., Yeganyan M. Interactive visual supports for children autism in Pers Ubiquit, 2010

[7] Android

Studio.

Online: https://developer.android.com/studio/index.html.

[8] Pivetta, E. M. Aplicação do software hot potatoes como ferramenta de apoio ao ensino/aprendizagem para pessoas com síndrome de Down. (2009).

[9] Leite D. A. \& Baptista N. M. G. Aprendizagem da criança de quatro a seis anos com Síndrome de Down em uma escola especial. (2006)

[10] Herrera, A. R. C., Dickie, I. B. \& Schulenburg, H. R. W. Design inclusivo: Interface Gráfica Voltada para Crianças com Síndrome de Down [electronic version]. (2014). Ergodesign \& HCI, 2, 1-8.

[11] Martin G. \& Pear J. Modificação de comportamento: o que é e como fazer [Translation Noreen Campbell de
Aguirre. Scientific Revision Hélio José Guilhard]. - 8. ed. - [Reprint]. (2009) - São Paulo: Roca.

[12] Douglas F. de M. Santos Edson, "ELViS: Enhanced for Limited Vision System.", International Journal of Applied Information Systems (IJAIS) - ISSN : 22490868 Foundation of Computer Science FCS, New York, USA Volume 9 - No.8, October 2015.

[13] C. J. Lee, "Externalizing and Interpreting Autonomic Arousal in People Diagnosed with Autism", Program of Media Arts and Sciences, School of Architetural and Planning in partial fulfillment of the requirements for the degree of Doctor of Philosophi, Massachusetts Institute of Technology, September 2011.

[14] Sommerville, Ian. Engenharia de Software. Tradução Selma Shin Shimizu Melnikoff; Reginaldo Arakaki; Edilson de Andrade Barbosa. 8. ed. São Paulo: Addison Wesley

[15] LEE, Chia-Hsun Jackie et al. Attention meter: a visionbased input toolkit for interaction designers. In: CHI'06 extended abstracts on Human factors in computing systems. ACM, 2006.

[16] Viola, P., and Jones Michael. "Robust Real-time Object Detection." Second International Workshop on Statistical and Computational Theories of Vision-Modeling, Learning, Computing, and Sampling. 2004.

[17] Belhumeur, Peter N.; Hespanha, João P.; KRIEGMAN, David J. Eigenfaces vs. fisherfaces: Recognition using class specific linear projection. IEEE Transactions on pattern analysis and machine intelligence, v. 19, n. 7, p. 711-720, 1997.

[18] I Tuytelaars T. Mikolajczyk K. Local Invariant Feature Detectors: A Survey, Foundations and Trends in Computer Graphics and Vision Vol. 3, No. 3. 2007

[19] Ramírez-Gutierrez, K., Cruz-Pérez, D., and PérezMeana, H. (2011). A face recognition algorithm using eigenphases and histogram equalization. International Journal of Computers, 5:34-41. 